\title{
Regulation Bancaire Et Prise De Risque Des Banques De La Cemac
}

\author{
Jean Francky Landry Ngono, \\ Université de Yaoundé II, Cameroon
}

Doi:10.19044/esj.2020.v16n1p122～URL:http://dx.doi.org/10.19044/esj.2020.v16n1p122

\section{Résumé}

Cet article évalue empiriquement l'effet de la régulation bancaire sur la prise de risques des banques dans la Communauté Economique et Monétaire de l'Afrique Centrale (CEMAC). Les données nécessaires pour réaliser cette étude proviennent de la Banque Mondiale, de la Banque des Etats de l'Afrique Centrale (BEAC) et de la Commission Bancaire de l'Afrique Centrale (COBAC). La période d'étude va de 2010 à 2017 et s'étend sur les six pays de la CEMAC. Afin d'atteindre cet objectif, les moindres carrés généralisés sont retenus à la suite du test de Hausman (1978). Il en ressort que les variations à la hausse de la régulation bancaire encouragent les banques à prendre plus de risques. En effet, les résultats montrent premièrement qu'une hausse du ratio de solvabilité conduit à une plus grande prise de risques des banques. Puis ces auteurs montrent qu'une hausse du ratio du capital réglementaire augmente la prise de risques par les banques. Néanmoins l'action des gouvernements de la région pour la promotion du secteur privé réduit la prise de risques des banques. Enfin, d'après ces résultats une politique monétaire expansionniste et une bonne conjoncture économique réduisent aussi la prise de risques des banques dans la CEMAC. Quelques recommandations de politiques économiques peuvent être faites à la lumière de ces résultats, notamment que les autorités en charge de la régulation bancaire dans la CEMAC doivent réduire le poids de la réglementation dans leur secteur bancaire puisque celleci semble ne pas être optimale pour réduire la prise de risques des banques. Egalement les gouvernements des différents pays de la région doivent accentuer leurs actions sur la promotion du secteur privé car elles contribuent à la réduction de la prise de risques par les banques dans la CEMAC.

Mots clés: Régulation bancaire, prise de risque, moindres carrés généralisés, politique monétaire 


\title{
Bank Regulation and Bank Risk-Taking in CEMAC
}

\author{
Jean Francky Landry Ngono, \\ Université de Yaoundé II, Cameroon
}

\begin{abstract}
This article empirically evaluates the effect of banking regulation on banks' risk taking in the Economic and Monetary Community of Central Africa (CEMAC). The data needed to carry out this study come from the World Bank, from "BEAC" and "COBAC". The study period is from 2010 to 2017 and covers the six CEMAC countries. In order to achieve this goal, generalized least squares are retained following the Hausman test (1978). It shows that upward variations in banking regulation encourage banks to take more risks. Indeed, the results show firstly that an increase in the solvency ratio leads to greater banks' risk taking. Then they show that an increase in the ratio of regulatory capital increases banks' risk taking. Nevertheless, the action of the governments of the region for the promotion of the private sector reduces the banks' risk taking. Finally, according to these results, expansionary monetary policy and good economic conditions also reduce banks' risk taking in CEMAC. Some economic policy recommendations can be made in the light of these results, notably that the authorities in charge of banking regulation in the CEMAC must reduce the weight of regulation in their banking sector since this seems not to be optimal to reduce banks' risk taking. Also the governments of the different countries of these region must emphasize their actions on the promotion of the private sector because they contribute to the reduction of the banks' risk taking in CEMAC.
\end{abstract}

Keywords: Bank regulation, generalized least-squares, monetary policy, risk taking

\section{Introduction}

La crise des subprimes de 2008 a remis à jour le problème de la prise de risques des banques (Borio et Zhu, 2012; Paligorova et Santos, 2017) c'està-dire l'incapacité de l'emprunteur à payer l'intérêt dû ou à rembourser le principal selon les termes spécifiés dans la convention de crédit (Greuning et Bratanovic, 2004). Les banques américaines ont accordé un volume élevé de crédits immobiliers à des ménages qui n'avaient pas des capacités de 
remboursements suffisantes. Cette prise de risques excessive de la part des banques a donné naissance à Bâle III (Ashraf et al. 2016).

Les pays de la Communauté Economique et Monétaire de l'Afrique Centrale (CEMAC), comme la plupart des économies en développement, n'ont pas été victimes de cette crise. Pourtant le rapport annuel de la Banque des Etats de l'Afrique Centrale (BEAC) de 2017 et le rapport annuel de la Commission Bancaire de l'Afrique Centrale (COBAC) de 2017 expliquent que ces dernières années les banques de la CEMAC ont pris plus de risques. Le rapport annuel 2017 de la COBAC considère ainsi que cette situation est préoccupante. Il convient alors de rechercher les éléments qui peuvent permettre de remédier à cette situation et au vu des travaux du comité de Bâle à la suite de la crise des subprimes sur la régulation bancaire, cette étude opte pour évaluer l'effet de la régulation bancaire sur la prise de risques des banques.

La régulation bancaire est « un ensemble de règles, d'incitations, et de pratiques des autorités publiques de supervision qui visent à instaurer et à maintenir la stabilité financière »(Mishkin, 2013). Et la théorie sur son effet sur la prise de risques des banques n'aboutit pas sur un consensus. Une partie de la littérature économique soutient que la prise de risques des banques peut être résorbée par une régulation optimale (Bolt et Tieman, 2004; Agoraki et al. 2011; Ashraf et al. 2016). Matutes et Vives (2000) expliquent que la restriction bancaire peut venir en complément de l'assurance dépôt pour une meilleure lutte contre la prise de risques des banques surtout lorsque la compétition entre ces dernières est élevée. Pollin (2005) pense que la régulation bancaire peut offrir au système bancaire une certaine stabilité. Tandis que d'autres auteurs pensent que celle-ci incite à un problème d'aléa moral et donc à une plus grande prise de risques par les banques (Merton, 1977; Bhattacharya et Thakor, 1993).

Ce manque de consensus se fait ressentir même dans les études empiriques. Agoraki et al. (2011) mènent une étude dans les pays d'Europe sur une période qui va de 1998 à 2005. En appliquant les generalized method of moments (GMM) de Blundell et Bond (1998) ces auteurs trouvent que la régulation bancaire conduit à la baisse la prise de risques bancaires dans ces pays. Toutefois ces auteurs montrent que la régulation bancaire seule ne permet pas de réduire la prise de risques des banques, elle trouve toute son efficacité en sa formulation en fonction du pouvoir de marché de chaque banque. Ashraf et al. (2016) qui mènent leur étude au Pakistan entre 2005 et 2012, utilisent à la fois la méthode des moindres carrés à variables indicatrices et la méthode des moments généralisés et trouvent que la régulation bancaire réduit la prise de risques des banques au Pakistan. Alors que González (2005) dans une étude sur 36 pays entre autre les Etats-Unis et l'Espagne, pour une 
période qui va de 1995 à 1999, trouve qu'une hausse de la régulation bancaire incite les banques à prendre plus de risques.

Cet article a pour objectif d'évaluer empiriquement l'effet de la régulation bancaire sur la prise de risques des banques dans la CEMAC. Pour ce faire les données de Banque Mondiale, de la BEAC et de la COBAC sont mobilisées sur une période qui va de 2010 à 2017. Ainsi grâce à l'application des moindres carrés généralisés, il en ressort une relation positive entre la régulation bancaire et la prise de risques des banques dans la CEMAC. Néanmoins, une politique monétaire expansionniste et une bonne conjoncture permettent de réduire cette prise de risques.

Le reste de cet article s'organise comme suit, le deuxième point porte sur une revue de littérature, le troisième sur la méthodologie, la quatrième sur les résultats et les discussions et le dernier point porte sur la conclusion et quelques recommandations de politiques économiques.

\section{Revue de littérature}

Le système bancaire a plusieurs fois montré sa capacité à connaitre des défaillances, soit à cause de problèmes internes, soit à cause de problèmes qui lui sont externes. Ces défaillances ont laissé percevoir le besoin de réglementer ce système et de s'assurer du respect de cette réglementation. Cette section permet de consulter la littérature sur l'effet de la régulation bancaire sur la prise de risques des banques.

\subsection{Assurance dépôt et prise de risques des banques}

En effectuant un dépôt à la banque, l'épargnant ne bénéficie que d'une rémunération fixe et faible. Toutefois en cas de faillite de la banque, l'épargnant perd les fonds mis à la disposition de la banque. De l'autre côté, la banque elle voit son profit augmenter lorsqu'elle investit dans des projets à rendements élevés qui sont aussi les plus risqués. Et en cas de faillite elle n'a qu'une responsabilité limitée. La banque a donc tendance à s'embarquer dans des projets risqués alors que les déposants ne l'approuvent pas (Jensen et Meckling, 1976). Afin de s'assurer de la bonne santé de leurs épargnes, les déposants cherchent à surveiller l'action de leur banque (Merton, 1977). Pourtant les déposants ne disposent pas de moyens pour contrôler l'action de la banque et encore moins pour la sanctionner (Dewatripont et Tirole, 1993; Merton, 1977; Scialom, 1999).

Dans l'optique de mettre en confiance les épargnants mais surtout de surveiller les banques, des mécanismes sont mis en place (Merton, 1977). L'un d'eux est l'assurance dépôts. Les banques ici sont tenues de contracter une assurance sur les dépôts qui sont faits en son sein. Ainsi en cas de faillite, l'assurance rembourse les dépôts que possédaient la banque (Diamond et Dybvig, 1983). L'assurance dépôts vient donc comme une première réponse 
valable pour résoudre le conflit entre la banque et les déposants (Black et Scholes, 1973; Merton, 1977). L'assurance dépôts elle-même s'avère limitée pour résoudre ce problème et oppose la littérature. D'un côté la littérature recense les biens faits de l'assurance dépôts, déjà parce qu'elle permet aux épargnants de rentrer dans leurs frais en cas de faillite, puis parce qu'elle assagit la banque. Pourtant d'un autre côté, la littérature postule que l'assurance dépôts donne à la banque la possibilité de prendre plus de risques puisqu'elle sait qu'en cas de faillite l'assurance dépôts paye les frais et en cas de réussite c'est elle qui bénéficie de la plus-value (Merton, 1977; Bhattacharya et Thakor, 1993; Greuning et Bratanovic, 2004; Pollin, 2005).

\subsection{Concentration de la propriété et prise de risques des banques}

Au-delà du conflit entre la banque et ses épargnants, il subsiste un autre conflit à l'intérieur même de la banque. Il s'agit du conflit entre les dirigeants et les actionnaires (Saunders et al. 1990; Hammami et Boubaker, 2015). L'intérêt des dirigeants divergeant parfois avec celui des actionnaires. Contrairement aux déposants, les actionnaires ont une possibilité de contrôle et de sanction sur la direction (Shleifer et Vishny, 1986; Unite et Sullivan, 2003; Garcia-Marco et Robles-Fernandez, 2008; Haw et al. 2010).

En effet, le problème des actionnaires tient prioritairement de faire du profit. Mais en tant qu'actionnaires, ce sont leurs fonds qui sont engagés dans la banque et par conséquent en cas de faillite ce sont eux qui subissent le plus. Ces éléments configurent donc le degré de prise de risques attendu des dirigeants. En ce qui concerne les dirigeants, tout dépend aussi du niveau d'engagement de leurs fonds dans la banque. Si les dirigeants ne sont que des employés qui n'ont aucun fond dans la banque, alors ces derniers ont tendance à protéger son emploi et ainsi à minimiser la prise de risques en se contentant d'assurer une certaine rémunération aux actionnaires (Amihud et Lev, 1981; Saunders et al. 1990). Par contre si les dirigeants ont faiblement engagés leurs fonds, leurs investissements vont se porter vers des projets risqués à rendements élevés question de se faire un maximum de profit. Enfin, si la direction a une grande participation en termes de capitaux dans la banque, la banque va s'engager dans des projets peu risqués car en cas de faillite la perte serait trop grande pour elle (Gorton et Rosen, 1995).

\subsection{Fonds propres réglementaires et prises de risques des banques}

La crise de 2008 a donné naissance à Bâle III, au canal du capital bancaire et au canal de la prise de risques. La régulation en place n'a pas permis de limiter la prise de risques des banques. C'est pourquoi la régulation bancaire aujourd'hui donne plus de poids à la réglementation en termes de fonds propres et que la littérature abonde autour du rôle joué par la politique monétaire. 
Les solutions précédentes ont révélé que plus la banque engage ses fonds, moins elle prend de risques. Ainsi, en lui imposant un minimum réglementaire de participation dans le capital, la banque investira moins dans des projets risqués. Les textes de Bâle ont rendu célèbre le ratio Cooke, qui établit le minimum réglementaire pour chaque banque en termes de fonds propres. L'objectif derrière une telle politique, entre autres, est de réduire la prise de risques des banques (Boot et Thakor, 1993; Kim et Santomero, 1994). Pourtant, la littérature ne s'accorde pas dessus.

En effet, une partie de la littérature postule que l'effet est le même que précédemment, c'est-à-dire que les banques ayant le minimum réglementaire de capital vont prendre plus de risques puisqu'elles savent qu'elles ont de quoi rembourser (Koehn et Santomero, 1980; Buser et al. 1981; Freixas et Rochet, 2008). D'un autre côté, des auteurs affirment que cette règle permet d'endiguer la prise de risques des banques. Plus ce minimum sera élevé moins les banques prendront des risques car elles ont trop à perdre (Boot et Thakor, 1993; Kim et Santomero, 1994, Agoraki et al. 2011). La littérature se concilie en ce qu'il faut un minimum optimal. Si la règle est optimale, pas assez forte pour asphyxier le système ou donner la confiance aux banques, pas assez faibles pour ne pas susciter la crainte de la perte chez les banques, alors le problème de la prise de risques peut être résolu.

Les études empiriques sur le sujet sont tout aussi éparses en termes de résultats. En effet, Altunbas et al. (2014) ont trouvé une relation positive entre les variations du capital et la prise de risques des banques en Europe. Alors que d'autres études comme celle de Jacques et Nigro (1997) aux Etats-Unis ou encore celle Konishi et Yasuda (2004) trouvent une relation négative entre le capital minimum et la prise des banques.

\subsection{Politique monétaire et prises de risques des banques}

L'effet de la politique monétaire sur la qualité des prêts accordés par les banques conduit à ce que Borio et Zhu (2012) appellent «Le Canal De La Prise De Risques ». Ce canal est défini par ces auteurs comme « L'impact des changements des taux directeurs sur la perception de risques ou la tolérance aux risques et donc sur le degré de risques des portefeuilles, sur le prix des actifs, sur les conditions des prix et d'extension du financement ». Ce canal opère de trois façons : par l'effet des taux d'intérêts sur les revenus et les flux de trésoreries des emprunteurs, par l'effet des taux d'intérêts sur la rentabilité des banques et enfin par la politique de communication et la fonction de réaction de la banque centrale.

La portée de ces éléments théoriques est si importante qu'aussi vite des auteurs ce sont mis à étudier ce lien empiriquement. Jiménez, Ongena, Peydró et Saurina (2014) effectuent une étude empirique dessus en Espagne. Jiménez et al. (2014) trouvent que lorsque les taux d'intérêts sont bas les banques 
accordent plus de prêts aux emprunteurs avec un mauvais historique, ou aucun historique, de crédit. Altunbas, Gambacorta et Marqués-Ibáñez (2014) mènent leur étude sur seize pays, dont quinze en Union Européenne et les Etats-Unis d'Amérique. Sur une période allant de 1998 à 2008 Altunbas et al. (2014) trouvent, en appliquant l'estimateur GMM, que de faibles niveaux des taux d'intérêts sur une longue période augmentent le risque bancaire.

Toutefois, dans la CEMAC des études dans ce sens sont presque inexistantes. De plus, la plupart d'entre elles ne cherchent pas à la fois l'effet de la régulation bancaire et de la politique monétaire sur la prise de risques des banques. Ce papier essaie justement de pallier à ces limites.

\section{Méthodologie}

Afin d'atteindre l'objectif que s'est fixé cette étude, il est nécessaire de recourir à des outils quantitatifs. Cette partie permet de présenter d'abord les variables et les sources des données, puis la spécification du modèle et enfin la méthode d'estimation.

\subsection{Variables et sources des données}

D’abord ce point présente les variables, puis leurs sources.

\section{-Prêt non performant}

Les travaux de Agoraki et al. (2011) utilisent le ratio des prêts non performants aux crédits bruts pour mesurer la prise de risques des banques. Cette étude cherchait à déterminer l'effet de la régulation et de la compétition sur la prise de risques des banques dans l'Europe de l'Est et dans l'Europe Centrale entre 1998 et 2005.

C'est cette même mesure qu'utilisent Ashraf et al. (2016). Ces derniers évaluent l'effet de la régulation du capital sur la prise de risques des banques. Hammami et Boubaker (2015) évaluent l'effet de la structure des fonds propres de la banque sur la prise de risques des banques de l'est et du nord de l'Afrique entre 2000 et 2010 et pour ce faire font aussi recours au rapport des prêts non performants et des crédits bruts.

Dans la même lignée que ces travaux, cette étude utilise également le rapport des prêts non performants aux crédits bruts afin de capter la prise de risques des banques dans la CEMAC.

\section{-Ratio de solvabilité et ratio de capital réglementaire}

Agoraki et al. (2011) étudient l'effet de la régulation et de la compétition sur la prise de risques des banques dans les pays d'Europe. Afin de mesurer la régulation bancaire, trois indices sont mobilisés. Un indice de capital réglementaire, un indice du pouvoir de supervision bancaire et un indice de restriction de l'activité bancaire. L'indice de capital réglementaire 
varie entre 0 et 8 . L'inde du pouvoir de supervision varie entre 0 et 12 . Et 1 'indice de restriction bancaire varie entre 1 et 4 .

Ashraf et al. (2016) dans leur étude au Pakistan cherchent l'effet de la régulation du capital sur la prise de risques des banques. Pour ce faire, dans leur étude la régulation du capital est mesurée par le ratio du capital. Cette même mesure est utilisée par Dannon et Lobez (2014) dans l'UEMOA. En effet, ces auteurs veulent évaluer de manière empirique l'efficacité de la régulation bancaire dans l'UEMOA. Afin de mesurer la régulation bancaire, ces auteurs recourent au ratio de solvabilité qui est égal au rapport du capital pondéré par le risque et le total d'actif et au ratio du capital réglementaire qui est égal au rapport du capital par le total d'actif. Ce sont ces deux derniers indicateurs qui sont utilisés dans ce travail.

\section{-Qualité de la régulation du gouvernement}

C'est la perception de l'habilité des gouvernements à formuler et à implémenter des règles pour promouvoir le secteur privé.

\section{-Taux d'intérêt d'appels d'offres}

Depuis la crise économique de 2008, une nouvelle littérature a émergé sur l'effet probable de la politique monétaire sur la prise de risques des banques. Borio et Zhu (2012) expliquent que la politique monétaire peut affecter la prise de risques des banques par trois groupes d'effets. De par son effet sur le rendement des banques, sur la situation patrimoniale des emprunteurs et de par l'effet de la politique de communication et de la fonction de réaction de la banque centrale sur les banques. C'est pourquoi cette étude introduit la politique monétaire comme variable explicative, tout comme Trinnou et Igue (2015), le principal taux directeur de la banque centrale est utilisé à savoir pour la BEAC le taux d'intérêt d'appels d'offres (TIAO).

\section{-Le taux de croissance du produit intérieur brut}

Et enfin, afin de capter l'effet de la conjoncture économique sur la prise de risques des banques, le taux de croissance du produit intérieur brut est aussi mobilisé.

\section{-Indice des prix à la consommation} CEMAC.

Cette variable permet de capter le taux d'inflation des pays de la

Les données nécessaires pour cette étude sont issues du rapport 2017 de la BEAC et du rapport 2017 de la COBAC pour ce qui du ratio de solvabilité et du ratio de capital réglementaire. Pour ce qui est du TIAO les données proviennent de la BEAC. Les données sur les prêts non performants, sur le taux de croissance du produit intérieur brut et sur le taux d'inflation 
proviennent de la base de données de la Banque Mondiale (World Development Indicators, 2018). Et les données sur la qualité de la régulation des gouvernements proviennent de (Worldwide Governance Indicators, 2018). La période d'étude, justifiée par la disponibilité des données, va de 2010 à 2017. La zone d'étude est la CEMAC, notamment le Cameroun, la République Centrafricaine, le Congo, le Gabon, la Guinée et le Tchad.

\subsection{Spécification du modèle}

Afin de déterminer l'effet de la régulation bancaire sur la prise de risques des banques, cette étude utilise le modèle de Ashraf et al. (2016). Ashraf et al. (2016) évaluent l'effet de la régulation du capital sur la prise de risques des banques au Pakistan. Soit alors la spécification suivante:

$P N P_{i, t}=\beta_{1} C O O K E_{i, t}+\beta_{2} C R_{i, t}+\beta_{3} T I A O_{i, t}+\beta_{4} Q R_{i, t}+\beta_{5} T P I B_{i, t}+$ $\beta_{6} I P C_{i, t}+n_{i}+\varepsilon_{i, t}$ avec $i=1, \ldots, 6$ et $t=2010, \ldots, 2017$

$P N P_{i, t}$ représente les prêts non performants des banques, soit précisément le rapport entre les prêts non performants et les crédits bruts, du pays i à la période t. $C O O K E_{i, t}$ représente le ratio de solvabilité des banques, c'est-à-dire le rapport entre le capital pondéré des risques et le total des actifs, du pays i à la période t. $C R_{i, t}$ représente le ratio du capital réglementaire des banques, il s'agit du rapport entre le capital bancaire et le total des actifs, du pays i à la période t. $T I A O_{i, t}$ représente le taux d'intérêt d'appels d'offres de la BEAC, c'est le principal taux directeur de la BEAC, à l'année t. $Q R_{i, t}$ représente la qualité de régulation des gouvernements, cette variable capte la perception de l'habilité des gouvernements à formuler et à implémenter des règles pour promouvoir le secteur privé, du pays i à la période t. TPIB $B_{i, t}$ le taux de croissance du produit intérieur brut, cet indicateur permet de capter la conjoncture économique, du pays i à la période t. $I P C_{i, t}$ représente le taux d'inflation, mesuré par l'indice des prix à la consommation, du pays i à la période t. $n_{i}$ représente l'effet spécifique individuel du pays i qui peut être fixe ou aléatoire et $\varepsilon_{i, t}$ représente le terme d'erreur. Puis $\beta_{1}, \beta_{2}, \beta_{3}, \beta_{4}, \beta_{5}$ et $\beta_{6}$ sont paramètres à estimer.

Tableau 1: Description des variables

\begin{tabular}{|l|l|l|}
\hline Variables & Description & Source des données \\
\hline PNP & $\begin{array}{l}\text { C'est le rapport entre les prêts non } \\
\text { performants et les crédits bruts }\end{array}$ & $\begin{array}{l}\text { World Development Indicators } \\
(2018)\end{array}$ \\
\hline COOKE & $\begin{array}{l}\text { Le ratio de solvabilité, il est égal au rapport } \\
\text { entre le capital pondéré des risques et le } \\
\text { total des actifs }\end{array}$ & $\begin{array}{l}\text { Rapports 2017 de la BEAC et de la } \\
\text { COBAC, Revue sur la Stabilité } \\
\text { Financière en Afrique Centrale de } \\
2016\end{array}$ \\
\hline CR & $\begin{array}{l}\text { Le ratio du capital réglementaire, il est égal } \\
\text { au rapport entre le capital bancaire et le total } \\
\text { des actifs }\end{array}$ & $\begin{array}{l}\text { Rapports 2017 de la BEAC et de la } \\
\text { COBAC, Revue sur la Stabilité } \\
\text { Financière en Afrique Centrale de } \\
2016\end{array}$ \\
\hline
\end{tabular}




\begin{tabular}{|l|l|ll|}
\hline TIAO & $\begin{array}{l}\text { Le taux d'intérêt des appels d'offres, } \\
\text { principal taux directeur de la BEAC, il capte } \\
\text { la politique monétaire de la BEAC }\end{array}$ & BEAC \\
\hline QR & $\begin{array}{l}\text { C'est la perception de l'habilité des } \\
\text { gouvernements à formuler et à implémenter } \\
\text { des règles pour promouvoir le secteur privé. }\end{array}$ & $\begin{array}{l}\text { Worldwide Governance Indicators } \\
(2018)\end{array}$ \\
\hline TPIB & $\begin{array}{l}\text { Le taux de croissance du produit intérieur } \\
\text { brut, il capte la conjoncture économique }\end{array}$ & $\begin{array}{l}\text { World Development Indicators } \\
(2018)\end{array}$ \\
\hline
\end{tabular}

Source: Auteur

\subsection{Méthode d'estimation}

La dimension individuelle est de 6 et la dimension temporelle est de 8, ainsi en ligne droite avec Ashraf et al. (2016), cette étude commence par effectuer le test de Hausman afin de choisir entre le modèle à effets spécifiques fixes et le modèle à effets spécifiques aléatoires. Selon le résultat du test de Hausman, il y'a une méthode d'estimation appropriée. Ainsi, si l'hypothèse nulle d'effets spécifiques aléatoires n'est pas rejetée alors la méthode d'estimation adéquate est les moindres carrés généralisés. Par contre si le modèle à effets spécifiques fixes est retenu les moindres carrés à variables indicatrices sont retenus comme dans l'étude de Ashraf et al. (2016).

\section{Résultats et discussions}

Cette section présente le résultat des tests de racines unitaires, du test de Hausman et des estimations.

\subsection{Résultats statistiques descriptives}

Cette section présente d'abord la relation linéaire qui existe entre la prise de risques et la régulation bancaire, respectivement le ratio de solvabilité et le ratio capital réglementaire, dans la CEMAC. Ensuite elle présente la matrice de corrélation de Pearson.

Figure 1. Relation linéaire entre la régulation bancaire et la prise de risques dans la CEMAC
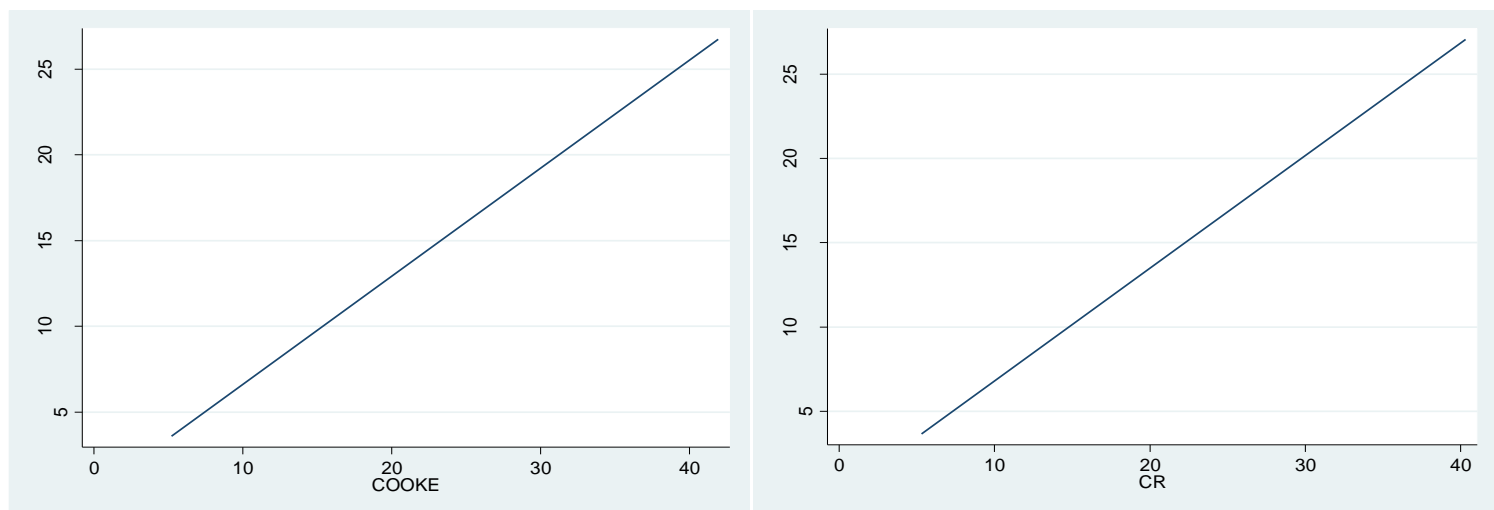

Source: Auteur sur Stata 14 
Les deux graphiques précédents montrent que la régulation bancaire a un effet positif sur la prise de risques des banques dans la CEMAC.

Tableau 2. La matrice de corrélation de Pearson

\begin{tabular}{|c|c|c|c|c|c|c|c|}
\hline & PNP & COOKE & CR & QR & TIAO & TPIB & IPC \\
\hline PNP & 1 & & & & & & \\
\hline COOKE & $0,6812^{*}$ & 1 & & & & & \\
\hline CR & $0,7201^{*}$ & $0,9523^{*}$ & 1 & & & & \\
\hline QR & $-0,4448^{*}$ & $-0,5001^{*}$ & $-0,5899^{*}$ & 1 & & & \\
\hline TIAO & $-0,3972^{*}$ & $-0,1582$ & $-0,1514$ & 0,1955 & 1 & & \\
\hline TPIB & $-0,4885^{*}$ & $-0,4541^{*}$ & $-0,4229^{*}$ & 0,2605 & 0,1475 & 1 & \\
\hline IPC & $0,4847^{*}$ & $0,5804^{*}$ & $0,5539^{*}$ & $-0,3617^{*}$ & $-0,2481$ & 0,0632 & 1 \\
\hline
\end{tabular}

Source: Auteur sur Stata 14

La matrice de corrélation de Pearson montre que le ratio COOKE et le ratio $\mathrm{CR}$ sont positivement corrélés avec la prise de risques des banques dans la CEMAC et ce lien est significatif au seuil de 5\%. Il ressort également que la régulation des gouvernements et le taux directeur de la BEAC sont négativement et significativement corrélés avec la prise des risques des banques tout comme le taux de croissance du produit intérieur brut. Enfin, il ressort que l'inflation est aussi positivement et significativement corrélée avec la prise de risques des banques. Le prochain point porte sur une analyse économétrique qui permet d'évaluer de manière robuste ce lien.

\subsection{Résultats des tests de racines unitaires}

Le premier point avant de procéder à une estimation est celui des tests de racines unitaires. Pour cela le test de Levin, Lin et Chu (LLC) est retenu ici car il fournit des informations sur la stationnarité de l'ensemble des individus du panel (Hurlin et Mignon, 2006).

Tableau 3. Les résultats du test de racine unitaire

\begin{tabular}{|c|c|c|}
\hline Variables $^{4}$ & Probabilité & Décision \\
\hline PNP & 0,0345 & $\mathrm{I}(0)$ \\
\hline COOKE & 0,0000 & $\mathrm{I}(0)$ \\
\hline CR & 0,0072 & $\mathrm{I}(0)$ \\
\hline TIAO & 0,0000 & $\mathrm{I}(0)$ \\
\hline QR & 0,0000 & $\mathrm{I}(0)$ \\
\hline TPIB & 0,0056 & $\mathrm{I}(0)$ \\
\hline IPC & 0,0000 & $\mathrm{I}(0)$ \\
\hline
\end{tabular}

Source: Auteur sur Stata 14

${ }^{4}$ Ces tests ont été effectués avec trend et constante sauf en ce qui concerne le TIAO qui est $\mathrm{I}(2)$ avec trend et constante et qui est fait juste avec la constante. Il est stationnaire en niveau au seuil de $1 \%$ lorsqu'on test sa stationnarité juste avec la constante et stationnaire à $5 \%$ lorsqu'on effectue ce test sans trend ni constante. 


\subsection{Résultats du test de Hausman}

Le test de Hausman (1978) permet d'opposer à l'hypothèse nulle d'effets aléatoires, l'hypothèse alternative d'effets fixes. Si la probabilité du test est inférieure à $10 \%$ alors l'hypothèse alternative n'est pas rejetée alors que si la probabilité est supérieure à $10 \%$ l'hypothèse nulle n'est pas rejetée.

\begin{tabular}{|l|l|l|l|l|l|l|l|l|}
\hline & Modèle 1 & Modèle 2 & Modèle 3 & Modèle 4 & Modèle 5 & Modèle 6 & Modèle 7 & Modèle 8 \\
\hline Probabilité & 0,9807 & 0,9931 & 0,9696 & 0,9573 & 0,9979 & 0,9985 & 0,9949 & 0,9838 \\
\hline Décision & H0 & H0 & H0 & H0 & H0 & H0 & H0 & H0 \\
\hline
\end{tabular}

Source: Auteur sur Stata 14

L'ensemble des modèles ont donné une probabilité supérieure à $10 \%$, ce qui signifie que c'est le modèle à effets aléatoires qui est retenu. Ainsi, la méthode d'estimation retenue est les moindres carrés généralisés. Cette méthode d'estimation produit des coefficients estimés consistants même en présence d'hétéroscédasticité et ou d'autocorrélation des termes d'erreurs.

\subsection{Résultats des estimations}

Le tableau suivant présente les résultats des estimations par la méthode des moindres carrés généralisés.

\begin{tabular}{|c|c|c|c|c|c|c|c|c|}
\hline & Modèle1 & Modèle2 & Modèle3 & Modèle4 & Modèle5 & Modèle6 & Modèle7 & Modèle8 \\
\hline COOK & \multirow{4}{*}{$\begin{array}{l}0,418^{* *} \\
(0,120)\end{array}$} & \multirow{4}{*}{$\begin{array}{l}0,600 * * * \\
(0,127)\end{array}$} & \multirow{4}{*}{$\begin{array}{l}0,455 * * * \\
(0,120)\end{array}$} & & \multirow{4}{*}{$\begin{array}{l}0,422^{* *} \\
(0,145)\end{array}$} & \multirow{4}{*}{$\begin{array}{l}0,557 * * * \\
(0,142)\end{array}$} & $-0,069$ & \multirow{2}{*}{$\begin{array}{l}0,046 \\
(0,221)\end{array}$} \\
\hline & & & & & & & $(0,206)$ & \\
\hline CR & & & & $0,597 * * *$ & & & $0,664 * *$ & $0,555^{* *}$ \\
\hline & & & & $(0,131)$ & & & $(0,235)$ & $(0,254)$ \\
\hline TIAO & \multirow{4}{*}{$\begin{array}{l}-4,449 * * \\
(1,100)\end{array}$} & \multirow{4}{*}{$\begin{array}{l}-4,043^{* * *} * \\
(1,020)\end{array}$} & $-3,473 * *$ & $-3,566^{* *}$ & \multirow{4}{*}{$\begin{array}{l}-3,330 * * \\
(1,169) \\
-7,541 * \\
(4,358)\end{array}$} & \multirow{4}{*}{$\begin{array}{l}-3,253^{* *} \\
(1,09) \\
-3,77 \\
(3,79)\end{array}$} & \multirow{4}{*}{$\begin{array}{l}-4,055^{* * *} \\
(1,035)\end{array}$} & \multirow{4}{*}{$\begin{array}{l}-3,504 * * \\
(1,105) \\
-4,773 \\
(4,011)\end{array}$} \\
\hline & & & $(1,151)$ & $(1,076)$ & & & & \\
\hline$\overline{Q R}$ & & & $-7,985 * *$ & $-4,295$ & & & & \\
\hline & & & $(3,840)$ & $(3,583)$ & & & & \\
\hline TPIB & \multirow{3}{*}{$\begin{array}{l}-0,236^{* *} \\
(0,091)\end{array}$} & \multirow{3}{*}{$\begin{array}{l}-0,239 * * \\
(0,082)\end{array}$} & $-0,236^{* *}$ & $-0,246^{* *}$ & \multirow{3}{*}{$\begin{array}{l}-0,263^{* *} \\
(0,100) \\
0,071 \\
(0,134)\end{array}$} & $-0,287 * *$ & \multirow{3}{*}{$\begin{array}{l}-0,245^{* *} \\
(0,085)\end{array}$} & \multirow{3}{*}{$\begin{array}{l}-0,243 \\
(0,083)\end{array}$} \\
\hline & & & $(0,087)$ & $(0,081)$ & & $(0,088)$ & & \\
\hline IPC & & & & & & $\begin{array}{l}0,123 \\
(0,113)\end{array}$ & & \\
\hline R-carré & 0,5976 & 0,6345 & 0,5375 & 0,6080 & 0,5536 & 0,6264 & 0,6379 & 0,6027 \\
\hline Fisher & 0,0000 & 0,0000 & 0,0000 & 0,0000 & 0,0000 & 0,0000 & 0,0000 & 0,0000 \\
\hline
\end{tabular}

Source: Auteur sur Stata 14

L'application des moindres carrés généralisés montre qu'il existe une relation positive entre la régulation bancaire et la prise de risques des banques dans la CEMAC. En effet, d'après le tableau qui précède plus le ratio de solvabilité des banques est élevé, ceteris paribus, plus celles-ci sont disposées à prendre des risques. Toutefois dans le modèle 07 ce ratio est négatif et non significatif, il se trouve que lorsque le ratio de solvabilité et le ratio de capital de réglementaire sont pris ensemble l'effet du ratio de solvabilité sur la prise de risques des banques devient non significatif. En ce qui concerne le ratio de 
capital réglementaire les résultats montrent que plus ce ratio est élevé, ceteris paribus, plus les banques prennent des risques.

Ces résultats s'alignent sur ceux de González (2005) et ceux de Agoraki et al. (2011). González (2005) trouve qu'une réglementation stricte conduit les banques à prendre plus de risques. C'est ce même résultat que trouvent Agoraki et al. (2011) qui évaluent l'effet de la régulation et de la compétition sur la prise de risques des banques. En effet, Claessens (2003) explique que théoriquement l'effet de la régulation bancaire sur la prise de risques des banques fait débat. Puisque d'un côté une régulation plus forte empêche les banques de tricher mais d'un autre côté elle empêche les banques de se diversifier ce qui permet de réduire la prise de risques (Agoraki et al. 2011). Toutefois la qualité de la régulation des gouvernements afin de promouvoir le secteur privé de la CEMAC a un effet négatif sur la prise de risques des banques, même si sa significativité ne tient que sur deux modèles.

En ce qui concerne les variables de contrôles, les résultats montrent qu'une politique monétaire expansionniste réduit la prise de risques des banques. Ce résultat s'accorde avec les travaux récents sur le canal de la prise de risques notamment ceux de Borio et Zhu (2012). Il ressort également qu'une meilleure conjoncture économique permet de réduire la prise des banques dans la CEMAC. Enfin ces résultats montrent que l'inflation n'a pas d'effet significatif sur la prise de risques des banques dans la CEMAC.

\section{Conclusion et recommandations de politique économique}

L'objectif de cette étude était d'évaluer l'effet de la régulation bancaire sur la prise de risques des banques dans la CEMAC sur une période qui va de 2010 à 2017. Les données utilisées proviennent de World Development Indicators (2018), de Worlwide Development Governance Indicators (2018), de la BEAC et de la COBAC. En appliquant les moindres carrés généralisés, cette étude trouve une relation positive entre la régulation bancaire et la prise de risques des banques dans la CEMAC. Il ressort également de cette étude que l'action des gouvernements pour promouvoir le secteur privé, ainsi qu'une politique monétaire expansionniste et une bonne conjoncture permettent de réduire la prise de risques des banques dans la CEMAC. Enfin, les résultats ont montré que le taux d'inflation de ces pays n'a pas d'effet significatif sur la prise de risques des banques.

D'après ces résultats, il est recommandé aux autorités chargées de la régulation bancaire dans la CEMAC de réduire le poids des réglementations sur les banques car celles-ci semblent ne pas être optimales afin de réduire la prise de risques des banques. Cette étude recommande également aux gouvernements des différents pays de la CEMAC d'accentuer leurs actions dans la réglementation et l'implémentation des règles afin de promouvoir le secteur privé car cela réduit la prise de risques des banques. Toutefois, les 
travaux de Agoraki et al. (2011) ont montré que si la régulation bancaire ne permet pas seule de réduire la prise de risques des banques, lorsqu'elle est faite selon le pouvoir de marché de chaque banque elle devient efficace dans ce sens. Cette étude n'a pas exploré ce point et cela peut servir d'axe de recherche pour de prochaines études.

\section{References:}

1. Agoraki, M-E., Delis, M. \& Pasiouras, F. (2011), «Regulations, Competition and Bank Risk-Taking in Transition Countries », Journal of Financial Stability, 7, pp. 38-48.

2. Altunbas, Y., Gambacorta, L. \& Marques-Ibáñez, D. (2014), « Does Monetary Policy Affect Bank Risk Taking? », International Journal of Central Banking, 10(1), pp. 95-136.

3. Amihud, Y.\& Lev. B. (1981), « Risk reduction as a managerial motive for merger, Bell Journal of Economics, 12, pp. 605-617.

4. Ashraf, B., Arshad, S. \& Hu, Y. (2016), « Capital Regulation and Bank Risk-Taking Behavior: Evidence from Pakistan », International Journal of Financial Studies, 4(16), pp. 1-20.

5. BEAC (2017), Rapport annuel sur l'exercice de l'année 2017

6. Bhattacharya, S. \& Thakor, A. (1993), «Contemporary banking theory », Journal of Financial Intermediation, 3, pp. 2-50.

7. Black, F. \& Scholes, M. (1973), « The pricing of options and corporate liabilities », Journal of Political Economy, vol. 81, n 3, pp. 637-654.

8. Blundell, R. \& Bond, S. (1998), «Initial Conditions and Moment Restrictions in Dynamic Panel Data Models », Journal of Econometrics, 87(1), pp. 115-143.

9. Bolt, W. \& Tieman, A. (2004), « Banking Competition, Risk and Regulation », Scandinavian Journal of Economics, 106(4), pp. 783804.

10. Boot, A. \& Thakor, A. (1993), « Self-interested bank regulation », American Economic Review, 83, pp. 206-212.

11. Borio, C. \& Zhu, H. (2012), « Capital Regulation, Risk-Taking and Monetary Policy: A Missed Link in the Transmission Mechanism? », Journal of Financial Stability, 8(5), pp. 236-251.

12. Buser, S., Chen, A. \& Kane, E. (1981), «Federal deposit insurance, regulatory policy, and optimal bank capital », The Journal of Finance, 36, pp. 51-60.

13. Claessens, S. (2003), « Benefits and costs of integrated financial services provision in developing countries ». In: Litan, R.E., Herring, R. (Eds.), Brookings-Wharton Papers on Financial Services. The Brookings Institution, Washington, pp. 85-139.

14. COBAC (2017), Rapport annuel sur l'exercice de l'année 2017 
15. Dannon, P. \& Lobez, F. (2014), « la régulation bancaire dans l'union économique et monétaire ouest-africaine est-elle efficace ? », Revue d'économie financière, 116, pp. 279-304.

16. Demirgüç-Kunt, A. \& Detragiache, E. (2002), « Does deposit insurance increase banking system stability? An empirical investigation ». Journal of Monetary Economics, 49, pp. 1373-1400.

17. Dewatripont, M. \& Tirole, J. (1993), La réglementation prudentielle des banques, Ed Payot Lausanne, Lausanne, pp. 1-177.

18. Diamond, D. \& Dybvig, P. (1983), « Bank Runs, Deposit Insurances, and Liquidity »The Journal of Political Economy, 91(3), pp. 401-419.

19. Freixas, X. \& Rochet, J. (2008), Microeconomics of banking, Second edition, The MIT Press, Cambridge, pp. 1-392.

20. Garcia-Marco, T. \& Robles-Fernández, M. (2008), « Risk-taking behaviour and ownership in the banking industry: The Spanish evidence », Journal of Economics and Business, (60)4, pp. 332-354.

21. Gonzalez, F. (2005), « Bank regulation and risk taking incentives: An international comparison of bank risk », Journal of Banking and Finance, 29, pp. 1153-1184.

22. Gorton G. \& Rosen R. (1995), « Corporate control, portfolio choice, and the decline of banking », The Journal of Finance, 50(5), pp. 13771420.

23. Greuning H. \& Bratanovic S. (2004), Analyse et gestion du risque bancaire : un cadre de référence pour l'évaluation de la gouvernance d'entreprise et $d u$ risque financier, traduction de Rozenbaum M., Edition Eska, Paris, pp. 1-384.

24. Hammami, Y. \& Boubaker, A. (2015), « Ownership Structure and Bank Risk-Taking: Empirical Evidence from the Middle East and North Africa », International Business Research, 8(5), pp. 271-284.

25. Hausman, J. (1978), « Specification Tests in Econometrics», Econometrica, 46(6), pp. 1251-1271.

26. Haw, M., Simon, H., Hu, B. \& Donghui, W. (2010), « Concentrated control, institutions and banking sector: An international study », Journal of Banking and Finance, 34, pp. 485-497.

27. Hurlin, C. \& Mignon, V. (2006), «Une Synthèse des Tests de Racine Unitaire sur Données de Panel », Economie et Prévision, Minefi Direction de la prévision, 169, pp. 253-294.

28. Jacques, K. \& Nigro, P. (1997), « Risk-based capital, portfolio risk, and bank capital: A simultaneous equations approach, », Journal Economics Business, 49, pp. 533-547.

29. Jensen, M. \& Meckling, W. (1976), «Theory of the firm: Managerial behavior, agency costs and ownership structure, Journal of Financial Economics, 13, pp. 305-60. 
30. Jiménez, G., Ongena, S., Peydró, J. \& Saurina, J. (2014), « Hazardous Times for Monetary Policy: What Do Twenty-Three Million Bank Loans Say About the Effects of Monetary Policy on Credit RiskTaking? », Econometrica, 82(2), pp. 463-505.

31. Kim, D. \& Santomero, A. (1994), "Risk in banking and capital regulation », The Journal of Finance, vol. 43, pp. 1219-1233.

32. Koehn, M. \& Santomero, A. (1980), « Regulation of bank capital and portfolio risk », The Journal of Finance, 35, pp.1235-1244.

33. Konishi, M. \& Yasuda, Y. (2004), « Factors affecting bank risk taking: Evidence from Japan », Journal of Banking and Finance, 28, pp. 215232.

34. Merton, R. (1977), «An analytic derivation of the cost of deposit insurance and loan guarantees », Journal of Banking and Finance, 1(2), pp. 3-11.

35. Mishkin F., (2013), The economics of money, banking and financial markets, $10^{\text {th }}$ edition, Pearson Education.

36. Pollin, J. (2005), « Des particularités de la gouvernance bancaire », in La nouvelle économie bancaire, (O. Pastré) Editions Economica, pp. 228-234.

37. Saunders, A., Strock, E. \& Travlos N. (1990), « Ownership Structure, Deregulation, and Bank Risk Taking », The Journal of Finance, 2, pp. 643-654.

38. Shleifer, A. \& Vishny, R. (1986), « Large shareholders and corporate control », Journal of Political Economy, 94, pp.461-488.

39. Scialom, L. (1999), Economie bancaire, Edition la découverte, Paris pp. 1-113.

40. Trinnou, C. \& Igue, C. (2015), « Risque De Crédit Bancaire Et Politique Monétaire Dans l'Union Economique Et Monétaire Ouest Africaine », Revue Economique et Monétaire de la BCEAO, 18, pp. 49-76.

41. Unite, A. \& Sullivan, M. (2003), «The effect of foreign entry and ownership structure on the Philippine domestic banking market ", Journal of Banking and Finance, 27, pp. 2323- 2345. 